\title{
Perfil socioeconômico, epidemiológico e farmacoterapêutico de idosos institucionalizados de Brasília, Brasil
}

\author{
The socio-economic, epidemiological and pharmaco-therapeutic \\ profile of institutionalized elderly individuals in Brasilia, Brazil
}

Mirna Poliana Furtado de Oliveira ${ }^{1}$ Maria Rita Carvalho Garbi Novaes ${ }^{1}$

${ }^{1}$ Programa de PósGraduação em Ciências da Saúde, Faculdade de Ciências da Saúde, Universidade de Brasília. Campus Universitário Darcy Ribeiro, Asa Norte. 90910-900 Brasília DF. mirnapoliana@hotmail.com

\begin{abstract}
The aim of this study is to describe the socioeconomic, epidemiological and pharmacotherapeutic profile of 154 elderly individuals from five homes for the aged in Brasilia using a questionnaire adapted from the Dader method and supplemented with information from medical records. The sample is made up of single people and widowers, mostly men, mean age of 74.6 years, with preserved cognition, low income and low education level. The group consumes between four and five medicines and is mainly affected by cardiovascular and psychiatric diseases. The drugs most used are cardiovascular and psychotropic medication. The adherence to pharmacotherapy is prejudiced by lack of knowledge about current medical prescription, difficulty of access and refusal to use prescribed medicines. Results suggest that this profile influences the quality and effectiveness of pharmacotherapy showing the need for pharmacotherapeutic follow-up for these patients as well as public health policies more focused on the specificities of the elderly to provide better quality of life and reduction of costs with health assistance.
\end{abstract}

Key words Elderly, Homes for the Aged, Health of institutionalized elderly individuals, Pharmaco-therapy, Use of medication
Resumo O objetivo deste estudo é descrever o perfil socioeconômico, epidemiológico e farmacoterapêutico de 154 idosos de cinco Instituições de Longa Permanência de Brasília por meio de questionário adaptado do Método Dáder e complementado com informações dos prontuários. A amostra é caracterizada por indivíduos solteiros e viúvos, em sua maioria homens, com idade média de 74,6 anos, sedentários, com estado cognitivo preservado, baixa renda mensal e baixo nivel de escolaridade. Ogrupo consome de quatro a cinco medicamentos e é acometido, principalmente, por distúrbios psiquiátricos e cardiovasculares. Os medicamentos mais utilizados são os cardiovasculares e os psicofármacos. A adesão à farmacoterapia é comprometida pela falta de conhecimento sobre a prescrição médica atual, dificuldade de acesso e recusa em tomar os medicamentos prescritos. Resultados sugerem que tal perfil influencia a qualidade e a eficácia da terapêutica medicamentosa indicando a necessidade de implementação de um acompanhamento farmacoterapeutico a esses idosos bem como de políticas públicas de saúde mais focadas nas particularidades dessa população a fim de proporcionar melhoria da qualidade de vida e redução dos custos com assistência à saúde.

Palavras-chave Idoso, Instituição de Longa Permanência para Idosos, Saúde do idoso institucionalizado, Farmacoterapia, Uso de medicamentos 


\section{Introdução}

O número crescente de idosos na população mundial e brasileira acarretou em uma alteração importante no perfil de morbimortalidade com predomínio das doenças crônico-degenerativas ${ }^{1}$. Doenças cardiovasculares, locomotoras e psiquiátricas são as mais prevalentes ${ }^{1-5}$ e exigem o uso continuo de medicamentos ${ }^{2,4,5}$. Estudos mostram que cada idoso toma em média de quatro a seis medicamentos ${ }^{2-7}$ e esse número é maior com o avanço da idade. Os medicamentos mais utilizados são os agentes cardiovasculares e os psicofármacos seguidos pelos antiinflamatórios, analgésicos e agentes gastrintestinais ${ }^{2,4,5,8}$.

$\mathrm{O}$ alto consumo de medicamentos entre essa população implica no crescimento do número de problemas relacionados a medicamentos (PRM) e da incidência de reações adversas ocasionando aumento das hospitalizações e dos custos com assistência à saúde $e^{4,5,7,9}$.

O envelhecimento populacional ocasionou ainda em mudanças socioeconômicas relevantes como aumento da demanda por serviços sociais e de saúde, antes características de países desenvolvidos ${ }^{1}$. Assim, fez-se necessária a reestruturação dos serviços de saúde aumentando a demanda pelos especializados de maior complexidade, além de capacitação da equipe envolvida na assistência a idosos. Nesse contexto, surgiram as Instituições de Longa Permanência de Idosos mantidas pelo governo, por associações religiosas e beneficentes ou pelos idosos e familiares, como opção para atender às necessidades sociais da sociedade moderna ${ }^{10}$.

A crescente procura por esses serviços é explicada, em muitos casos, pelas dificuldades econômicas e psicossociais encontradas pelas famílias para o cuidado do idoso; sobretudo para aqueles que apresentam redução da capacidade funcional ${ }^{10}$. Tais idosos tornam-se cada vez mais dependentes para realização de atividades cotidianas e requerem cuidados de maior complexidade e custo ${ }^{10}$.

Em alguns casos, a institucionalização pode representar rompimento do convívio familiar, abandono e isolamento social. Acredita-se que essa situação aliada às condições de saúde, à carência de profissionais capacitados e à dificuldade de acesso desse grupo aos serviços públicos de saú$\mathrm{de}^{11}$, influenciam no acesso e na adesão à farmacoterapia $^{6,12}$, bem como no surgimento e agravo dos PRM. Além disso, a exclusão dos idosos institucionalizados das políticas públicas de saúde existentes no Brasil ${ }^{11}$ agravam a situação de saúde desse grupo e a incidência desses problemas.
Nessa perspectiva, este trabalho tem como objetivo descrever o perfil socioeconômico, epidemiológico e farmacoterapêutico de idosos de Instituições de Longa Permanência de Brasília a fim de determinar as principais variáveis que influenciam a qualidade da terapêutica medicamentosa e de fornecer subsídios para a (re)estruturação de políticas de saúde voltadas para as necessidades particulares dessa população, em constante crescimento.

\section{Métodos}

Estudo epidemiológico, transversal, descritivo e exploratório realizado com idosos residentes em cinco instituições de longa permanência do Distrito Federal. Os dados foram coletados no período de janeiro a dezembro de 2007. A amostra, obtida por conveniência, foi constituída por 154 indivíduos, de ambos os sexos, com idade igual ou superior a 60 anos e que consumiam pelo menos um medicamento de uso contínuo. A proporção total de idosos excluídos do estudo por não se enquadrar em tal perfil foi de apenas seis indivíduos (2,0\% da população total). Foram incluídas as instituições ativas no cadastro do Conselho dos Direitos do Idoso do Distrito Federal e que aceitaram participar voluntariamente da pesquisa. Todas as instituições participantes são filantrópicas, possuindo um total de 300 idosos. Os participantes foram classificados em dois grupos: com e sem discernimento, baseado na capacidade de fornecer e receber informações corretas e coerentes. Tal classificação foi feita pela própria equipe da instituição.

Os dados foram coletados através de um questionário adaptado do Método Dáder, específico para acompanhamento farmacoterapêutico a pacientes ambulatoriais criado por pesquisadores espanhóis ${ }^{13}$. O questionário avaliou as cinco primeiras fases propostas pelo método de seguimento farmacoterapêutico (oferta do serviço, primeira entrevista, análise da situação, fase de estudo e avaliação) considerando as peculiaridades da população estudada e as limitações para a coleta dos dados. Por ser institucionalizada, a população estudada apresenta várias características importantes tais como limitação de acesso aos serviços de saúde e de convívio social; acompanhamento por número limitado de profissionais, que em geral são voluntários; ausência de autonomia em relação aos medicamentos utilizados, uma vez que a administração ocorre sob supervisão dos cuidadores e nos horários 
estabelecidos por eles de acordo com a prescrição médica; e carência de informações nos prontuários médicos presentes nas instituições.

Outra característica importante da amostra é a relação socioeconômica dos idosos com as instituições. Os pacientes contribuem com um valor financeiro mensal irrisório (30\% do salário mínimo na época da pesquisa) para colaborar no custeio da manutenção das instituições, porém, as mesmas possuem caráter filantrópico e recebem doações da comunidade. O restante da renda mensal do idoso é utilizado para compra de objetos pessoais (roupas e produtos de higiene), de alimentos e medicamentos que eventualmente faltam nas instituições. Grande parte dos medicamentos utilizados pelos idosos é obtida na rede pública de saúde do Distrito Federal e somente são adquiridos os medicamentos que não são de distribuição gratuita pelo SUS ou que estão em falta. Para os idosos, sem renda mensal, a instituição garante a disponibilidade de tais medicamentos com os recursos que recebem.

Os dados referentes a custo mensal com medicamentos foram obtidos a partir de informações das próprias instituições, que mantêm anotações do controle financeiro da renda de cada idoso. Para aqueles cuja renda mensal é gerenciada pela família, as instituições possuem informações apenas dos medicamentos que foram comprados por recursos próprios. Para esses idosos, a renda mensal foi informada pelo idoso com discernimento ou pelo cuidador e o gasto com medicamentos foi obtido por verificação do preço dos mesmos no mercado, no momento da pesquisa e o percentual equivalente da renda média mensal informada.

O questionário foi respondido pelos idosos considerados com discernimento e pelos cuidadores daqueles considerados sem discernimento. As informações obtidas nas entrevistas foram complementadas com análise dos prontuários e prescrições médicas.

As variáveis analisadas foram: Perfil socioeconômico (sexo, idade, escolaridade, renda mensal, estado civil, tempo de institucionalização, percepção quanto à institucionalização e convívio familiar), Perfil epidemiológico (doenças prevalentes), prática de atividades físicas e Perfil Farmacoterapêutico (medicamentos utilizados, número de medicamentos por idoso, custo mensal com medicamentos, nível de conhecimento do idoso quanto a nome, indicação e posologia dos medicamentos utilizados, fatores de não adesão ao tratamento e acesso a medicamentos). Os medicamentos foram classificados segundo a
Anatomical-Therapeutical-Chemical Classification System (ATC).

Os dados referentes à prática de atividades físicas foram fornecidos pelos próprios idosos e pelos cuidadores considerando sedentários os acamados e os idosos que não realizam nenhuma atividade física semanalmente. Foram considerados para o cálculo dessa variável, os idosos que realizam qualquer atividade física pelo menos uma vez por semana nos últimos seis meses. Em quatro das instituições (80,0\%) existem programas de combate ao sedentarismo incluindo aulas de natação e hidroginástica ministradas por educadores físicos, além de que alguns idosos, em geral os de autonomia preservada, fazem caminhadas pela instituição ou em seus arredores.

O grau de autonomia para realização de atividades diárias foi definido pelas equipes das instituições; baseado nos resultados do Teste de Avaliação da Capacidade Funcional segundo a Escala de Katz (citado por Araújo e Ceolim) ${ }^{14}$ aplicado por educadores físicos, parceiros do projeto de pesquisa e voluntários nas instituições pesquisadas. Tal teste foi aplicado sistematicamente nas cinco instituições utilizando-se os mesmos parâmetros.

Os dados referentes à percepção em relação à institucionalização foram obtidos a partir do questionamento direto ao idoso com discernimento, que avaliou a frequência (frequentemente, às vezes, raramente ou nunca) em que se sente deprimido e ansioso e os sintomas mais comuns de tais situações (tristeza, desânimo, descrença, isolamento, choro, tentativa de suicídio, ideia de morte, agitação, insônia, palpitações, sudorese). Além disso, os idosos com discernimento foram indagados quanto ao sentimento em relação à instituição onde vive (satisfeito ou não satisfeito).

A prevalência de depressão e distúrbios psiquiátricos na amostra foi contabilizada considerando os casos diagnosticados por profissionais habilitados (psicólogos e psiquiatras) e que constavam nos prontuários das instituições; não sendo confirmado por meio da aplicação de testes específicos pelos pesquisadores além de não se obter informações exatas da existência de sistematização para diagnóstico nas cinco instituições. Ressalta-se que todas as instituições são providas de psicólogos e psiquiatras. Além destes, também possuem, em numero limitado, assistência de médicos, enfermeiros, fisioterapeutas, terapeutas ocupacionais e nutricionistas. Nenhuma instituição possui farmacêutico responsável.

Os dados foram alocados em SPSS (Statistical Package for Social Sciences) versão 15.0 e ana- 
lisados utilizando os testes Qui-quadrado e t de student, considerando os resultados significativos para $\mathrm{p}<0,05$. As associações entre variáveis foram avaliadas através de sequências de modelos log-lineares aninhados: de associação homogênea, de independência condicional e de independência conjunta. Para testar o efeito da ausência de associações entre as variáveis para a sequência de modelos, utilizou-se o teste condicional de Qui-quadrado da razão de verossimilhança com nível de significância de 5\%.

O protocolo da pesquisa foi aprovado pelo Comitê de Ética em Pesquisa da Secretaria de Estado de Saúde do Distrito Federal. Os participantes que aceitaram participar voluntariamente da pesquisa assinaram o Termo de Consentimento Livre e Esclarecido e as instituições o Termo de Concordância.

\section{Resultados}

Dos 154 idosos, 51,3\% eram do sexo masculino e $53,9 \%$ foram considerados com discernimento. Entre os idosos sem discernimento, as mulheres constituíram a maioria $(\mathrm{p}=0,0164)$. Os idosos apresentaram idade média de 74,6 anos e vivem nas instituições há cerca de 5,4 anos, não havendo diferença significativa entre os sexos para tais variáveis. Foi observada ainda, maior prevalência de idosos com idade superior a 75 anos em relação aos demais grupos etários.

Quanto ao nível de instrução, 46,6\% não possuíam nenhuma instrução ou estudaram menos de 1 ano e 43,9\% dos idosos possuíam formação primária tendo estudado até 7 anos. A maioria no grupo possuía renda mensal média de até 2 salários-mínimos $(\mathrm{R} \$ 830,00)$ e era constituído por solteiros e viúvos sendo que para nenhum desses dados houve diferença significativa entre os sexos (Tabela 1).

A maioria dos idosos com discernimento $(83,2 \%)$ apresentou independência para realizar atividades diárias como tomar banho, vestir-se e caminhar pela instituição, ao contrário da maioria dos considerados sem discernimento $(80,3 \%)$ que depende dos cuidadores $(\mathrm{p}<0,0001)$ para tais atividades.

Quando indagados sobre a auto percepção em relação à institucionalização; $66,3 \%$ dos idosos com discernimento, disseram gostar da instituição sendo que 55,4\% relataram não sentir-

Tabela 1. Dados socioeconômicos de idosos de instituições de longa permanência de Brasília.

\begin{tabular}{|c|c|c|c|c|c|c|}
\hline \multirow{2}{*}{ Variável } & \multicolumn{2}{|c|}{ Mulheres } & \multicolumn{2}{|c|}{ Homens } & \multicolumn{2}{|c|}{ Total } \\
\hline & $\mathbf{n}$ & $\%$ & $\mathbf{n}$ & $\%$ & $\mathbf{n}$ & $\%$ \\
\hline \multicolumn{7}{|l|}{ Faixa etária } \\
\hline 60-64 anos & 14 & 18,67 & 12 & 15,19 & 26 & 16,88 \\
\hline 65-69 anos & 11 & 14,67 & 18 & 22,78 & 29 & 18,83 \\
\hline 70-74 anos & 11 & 14,67 & 12 & 15,19 & 23 & 14,94 \\
\hline$>75$ anos & 39 & 52,00 & 37 & 46,84 & 76 & 49,35 \\
\hline Total & 75 & & 79 & & 154 & 100,00 \\
\hline \multicolumn{7}{|l|}{ Estado Civil } \\
\hline Solteiro & 33 & 44,59 & 37 & 48,68 & 70 & 46,67 \\
\hline Viúvo & 28 & 37,84 & 20 & 26,32 & 48 & 32,00 \\
\hline Separado/Divorciado & 13 & 17,57 & 19 & 25,00 & 32 & 21,33 \\
\hline Total & 74 & & 76 & & 150 & 100,0 \\
\hline \multicolumn{7}{|l|}{ Escolaridade $^{\mathrm{a}}$} \\
\hline Nenhuma & 38 & 52,05 & 31 & 41,33 & 69 & 46,62 \\
\hline 1 a 7 anos & 30 & 41,10 & 35 & 46,67 & 65 & 43,92 \\
\hline 8 a 10 anos & 03 & 4,11 & 08 & 10,67 & 11 & 7,43 \\
\hline$>10$ anos & 02 & 2,74 & 01 & 1,33 & 03 & 2,03 \\
\hline Total & 73 & & 75 & & 148 & 100,00 \\
\hline \multicolumn{7}{|c|}{ Renda média mensal $\left(\mathrm{SM}^{\mathrm{b}}\right)$} \\
\hline$<2$ & 48 & 64,00 & 64 & 81,01 & 112 & 72,73 \\
\hline $2-3$ & 23 & 30,67 & 10 & 12,66 & 33 & 21,43 \\
\hline$>4$ & 04 & 5,33 & 05 & 6,33 & 09 & 5,84 \\
\hline Total & 75 & & 79 & & 154 & 100,00 \\
\hline
\end{tabular}

a Escolaridade (por anos de estudo). ${ }^{\text {b }}$ SM (salário-mínimo) vigente à época da pesquisa (em reais) $=\mathrm{R} \$ 415,00$ 
se deprimidos e $67,5 \%$ não se queixaram de ansiedade, sem diferença significativa entre os sexos para tais variáveis. Em relação ao convívio familiar e à institucionalização, foi observado que apenas 51,0\% ( $\mathrm{n}=78)$ dos idosos recebem visita de seus familiares sendo que a proporção não difere entre os sexos $(p=0,4209)$.

Quanto à prática de atividades físicas, a maioria dos idosos foi considerada sedentária $(79,5 \%)$ não havendo diferença significativa entre sexos $(p=0,2111)$.

O grupo é acometido, principalmente, por problemas de saúde do sistema cardiovascular $(82,5 \%)$ e nervoso $(54,6 \%)$, sendo os mais prevalentes: hipertensão arterial sistêmica $(76,6 \%)$, problemas articulares $(16,3 \%)$, diabetes mellitus tipo II (16,2\%), problemas respiratórios (13,0\%), insuficiência cardíaca $(12,3 \%)$ e problemas gastrintestinais $(11,7 \%)$. Os idosos com discernimento são acometidos por um número maior de problemas de saúde que os sem discernimento $(\mathrm{p}<0,0001)$.

Análise estatística apontou uma associação positiva entre nível de satisfação com a instituição e incidência de depressão e doenças psiquiátricas entre os idosos $(\mathrm{p}<0,001)$. No grupo estudado, os idosos que referiram não estar satisfeitos com a instituição e com a situação como vivem, queixando-se de sintomas como ansiedade, tristeza e isolamento $(33,7 \%)$, são portadores de distúrbios de humor, como depressão; ou distúrbios psiquiátricos (33,7\%); sendo que os idosos que disseram gostar da instituição são menos suscetíveis à depressão $(\mathrm{p}<0,0001)$. Tendência semelhante foi observada quando analisada associação entre casos de distúrbios psiquiátricos e nível de satisfação com a institucionalização $(\mathrm{p}<0,0001)$.

No grupo estudado, o número de medicamentos de uso contínuo consumidos pelas mulheres foi superior ao dos homens ( $p<0,0001)$. Em média cada idoso $(\mathrm{n}=79)$ tomava 4,16 medicamentos (DP 2,23) enquanto as idosas $(\mathrm{n}=$ 75) tomavam 8,96 medicamentos (DP 3,39).

Os medicamentos mais utilizados pelos idosos foram os agentes cardiovasculares $(81,2 \%)$, do sistema nervoso central $(63,0 \%)$, do sistema endócrino $(18,9 \%)$, do sistema gastrintestinal $(16,9 \%)$ e do sistema respiratório $(7,8 \%)$.

$\mathrm{O}$ estudo mostrou ainda que os idosos com discernimento consumiam mais medicamentos que os sem discernimento (cinco e quatro medicamentos, respectivamente) $(\mathrm{p}=0,008)$, sendo que o consumo de medicamentos foi maior entre as mulheres com discernimento $(p<0,0001)$.
Foi verificado ainda, que 98,2\% dos idosos utilizavam um psicofármaco sendo que os antipsicóticos (29,9\%), hipnóticos, sedativos e ansiolíticos $(24,0 \%)$, antidepressivos $(22,1 \%)$ e anticonvulsivantes $(14,3 \%)$ foram os mais consumidos. Apenas os antipsicóticos foram mais consumidos pelas mulheres $(\mathrm{p}=0,041)$ não havendo diferença significativa entre os sexos para as demais classes.

Análise estatística mostrou associação positiva entre número de medicamentos utilizados e idade $(\mathrm{p}=0,045)$ sendo que os idosos entre $60 \mathrm{e}$ 64 anos consumiam de 1 a 3 medicamentos ( $\mathrm{p}=$ $0,0091)$ e o consumo foi maior entre os idosos de idades mais avançadas, tendência observada em ambos os sexos (Figuras 1 e 2).

Acerca do nível de informação do idoso com discernimento $(\mathrm{n}=83)$ sobre os medicamentos utilizados no momento da pesquisa, foi observado que $69,9 \%$ tiveram alguma recordação da prescrição médica e as mulheres souberam dar mais informações sobre nome, indicação e posologia dos medicamentos utilizados que os homens. No grupo, 40,0\% dos homens não souberam dar nenhuma informação sobre a terapia medicamentosa e esse número foi menor entre as mulheres (15,2\%) (Tabela 2).

Os dados mostraram que $92,8 \%$ dos idosos com discernimento tomavam seus medicamentos sozinhos, porém sob supervisão dos cuida-

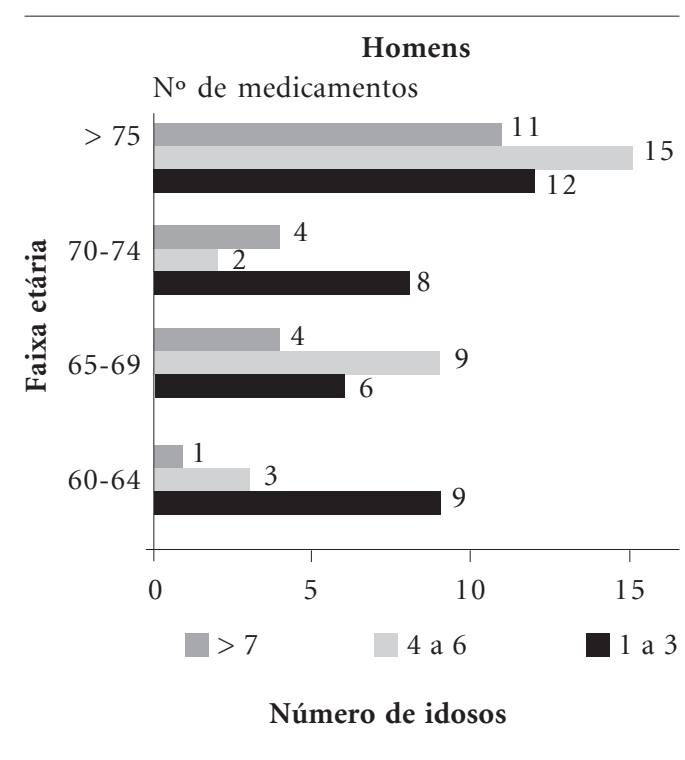

Figura 1. Relação entre idade e número de medicamentos utilizados por idosos do sexo masculino de Instituições de Longa Permanência de Brasília $(\mathrm{n}=79)$ 


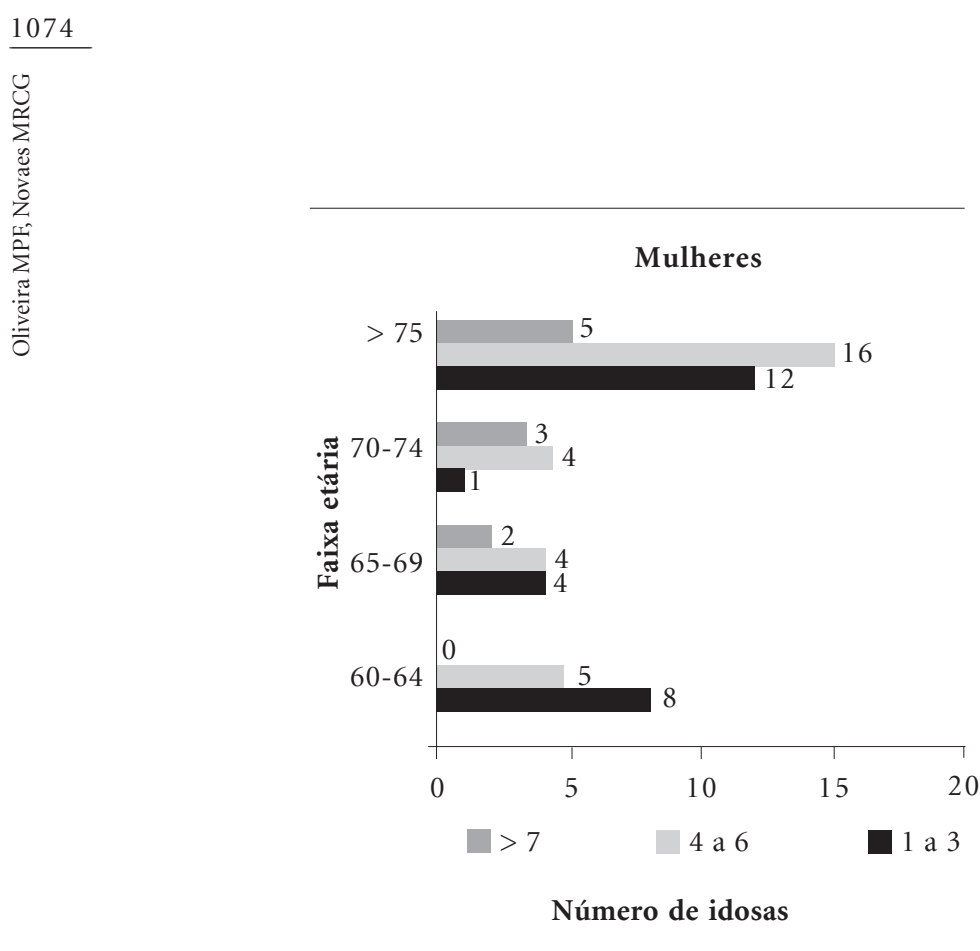

Figura 2. Relação entre idade e número de medicamentos utilizados por idosos do sexo feminino de Instituições de Longa Permanência de Brasília $(\mathrm{n}=75)$

Tabela 2. Nível de conhecimento de idosos com discernimento sobre a prescrição médica atual $(\mathrm{n}=83)$

\begin{tabular}{lcc}
\hline \multicolumn{1}{c}{ Variável } & $\begin{array}{c}\text { Homens } \\
(\%)\end{array}$ & $\begin{array}{c}\text { Mulheres } \\
(\%)\end{array}$ \\
\hline $\begin{array}{l}\text { Souberam dar alguma } \\
\text { informação }\end{array}$ & 60,0 & 84,8 \\
$\begin{array}{l}\text { Informação correta sobre } \\
\text { nomes }\end{array}$ & 43,4 & 42,2 \\
$\begin{array}{l}\text { Informação correta sobre } \\
\text { indicação }\end{array}$ & 41,0 & 37,9 \\
$\begin{array}{l}\text { Informação correta sobre } \\
\text { posologia }\end{array}$ & 36,5 & 49,0 \\
\hline
\end{tabular}

dores das instituições; enquanto 56,3\% entre os sem discernimento dependiam dos cuidadores para administrá-los utilizando os medicamentos, em geral, por via parenteral ou enteral (sonda). Os demais idosos sem discernimento $(43,7 \%)$ possuíam capacidade de deglutição preservada e portanto de tomar os medicamentos sozinhos, sob supervisão dos cuidadores.

$28,6 \%$ dos idosos com discernimento e $29,3 \%$ dos sem discernimento apresentaram algum problema que comprometiam a adesão à terapia medicamentosa. Os principais problemas identificados foram: dificuldade de acesso aos medi- camentos prescritos $(61,7 \%)$, recusa em tomar o medicamento $(48,3 \%)$, sensação de desconforto gastrintestinal (18,9\%), dificuldade de deglutição $(10,1 \%)$, queixa de gosto ou cheiro ruim e/ou administração incômoda $(2,3 \%)$.

$\mathrm{O}$ acesso do grupo a medicamentos foi avaliado pela disponibilidade dos prescritos nas próprias instituições e nos centros de saúde do Distrito Federal próximos às mesmas considerando a Relação de Medicamentos Essenciais do Distrito Federal (REME). Nas instituições pesquisadas, 96,5\% dos medicamentos prescritos para os idosos $(\mathrm{n}=154)$ estavam disponíveis no momento da pesquisa e $80,0 \%$ dos mesmos estavam disponíveis na rede de Atenção Básica à Saúde do Distrito Federal. O gasto com os medicamentos não disponíveis totalizava $12,3 \%$ da renda média mensal de cada idoso $(\mathrm{p}=0,025)$.

\section{Discussão}

Os resultados deste estudo corroboram dados mostrados por outros trabalhos referentes ao perfil socioeconômico, epidemiológico e farmacoterapêutico da população idosa brasileira ${ }^{2-5,8,15-20}$.

Apesar da existência de inúmeros trabalhos mostrando que a expectativa de vida entre as mulheres é maior que nos homens e, por conseguinte, maior proporção das mesmas na população idosa ${ }^{3,4,6,8,19}$; devido a uma maior proteção cardiovascular fornecida pelos hormônios femininos, menor consumo de álcool e tabaco e maior procura por assistência médica ${ }^{6}$; no grupo estudado não foi encontrada diferença significativa de idade entre os sexos.

Resultados apontaram ainda um envelhecimento da própria população idosa (Tabela 1) devido à maior prevalência de idosos acima de 75 anos; também mostrado por Chaimowicz ${ }^{1}$ e Castellar et al. ${ }^{15}$ indicando aumento crescente da expectativa de vida na população brasileira.

O aumento do número de idosos na população ocasionou em mudanças socioeconômicas e culturais relevantes ${ }^{1}$. Na sociedade moderna, muitas famílias não apresentam condições de cuidar de seus idosos, aumentando a procura pelas Instituições de Longa Permanência ${ }^{10}$. Estudos mostram que a dependência crescente que os idosos apresentam para realizar atividades rotineiras, o despreparo da maioria das famílias para cuidar de seus idosos, o elevado número de doenças e de medicamentos utilizados aliados à baixa renda familiar estão entre as causas de institucionalização ${ }^{10,17}$. 
O presente estudo sugere que tais fatores podem ser causa da institucionalização do grupo estudado constituído, sobretudo por idosos sozinhos (solteiros ou viúvos), de baixo nível de instrução, baixa renda mensal, sem convívio familiar, com um grau de dependência significativo, um número crescente de problemas de saúde, usuários de polifarmácia e com gastos crescentes com assistência à saúde. Corroborando tal hipótese, uma pesquisa realizada com idosos longevos em Minas Gerais mostrou associação significativa entre idade, polifarmácia, afastamento do convívio familiar e social e capacidade funcional. Segundo os autores, quanto maior o consumo de medicamentos e menor o convívio familiar, maior o comprometimento da capacidade funcional do idoso e tal situação é agravada com o avanço da idade ${ }^{21}$.

Ainda com a possibilidade de que a institucionalização pode representar para o idoso um rompimento dos laços familiares e isolamento social gerando solidão, depressão, desânimo, descrença e transtornos psíquicos, a maior parte dos idosos deste estudo declarou que gosta da instituição onde vive e não se queixa de tristeza ou ansiedade patológica. Apesar disso, análise das prescrições médicas mostrou um alto consumo de psicofármacos, sendo os antidepressivos e os antipsicóticos os mais prescritos indicando uma prevalência significativa de transtornos afetivos e psiquiátricos no grupo estudado. Tais dados podem sugerir aceitação pelo idoso da própria situação, desesperança em relação ao futuro e uma supermedicalização do grupo avaliado. Além disso, a elevada prescrição de psicofármacos para idosos pode refletir um despreparo dos prescritores, dos serviços de saúde e da própria sociedade para lidar com os idosos e as peculiaridades que acompanham o envelhecimento, sendo necessário medicá-los para tratar situações comuns da idade como insônia, ansiedade e quadros depressivos.

Estudos mostram o uso crônico de psicofármacos por idosos, sobretudo os hipnóticos, os sedativos e os ansiolíticos da classe dos benzodiazepínicos, devido à constante insônia e ansiedade que acometem o grupo com graves consequências como aumento das interações medicamentosas, reações adversas, desenvolvimento de dependência e tolerância ${ }^{21}$.

Segundo Cruz et al. ${ }^{22}$, a insônia acomete mais as mulheres, pessoas divorciadas, viúvas e aquelas com menor grau de escolaridade e baixa renda salarial, situações observadas no grupo estudado.

O perfil epidemiológico do grupo estudado já fora mostrado por outros estudos que apon- taram as doenças cardiovasculares, neurológicas, psiquiátricas e ortopédicas como as mais prevalentes nessa faixa etária ${ }^{2,4,5,8,15,18-20}$.

Acredita-se que tal perfil de morbidade aliado ao sedentarismo do grupo estudado e ao elevado consumo de medicamentos, sobretudo de psicofármacos, podem ser causas de redução da capacidade funcional e conseguinte baixa qualidade de vida desses idosos.

Nogueira et al. ${ }^{21}$ mostrou uma associação positiva relevante entre número de medicamentos utilizados e capacidade funcional em idosos longevos. Segundo os autores, quanto maior a utilização de medicamentos, maior o comprometimento da capacidade funcional dos usuários ${ }^{21}$, provavelmente devido a interações medicamentosas e potencialização de seus efeitos adversos.

A maior prevalência de doenças e, por conseguinte, o maior consumo de medicamentos entre os idosos considerados com discernimento pode refletir uma melhor capacidade desse grupo em se comunicar e se expressar quando comparados aos idosos considerados sem discernimento. Os problemas de saúde entre idosos com comprometimento cognitivo podem passar despercebidos aos profissionais de saúde porque os pacientes não conseguem descrever seus sintomas adequadamente ${ }^{19}$. Além disso, os idosos com discernimento podem ser "poliqueixosos" comparados ao sem.

A maior prevalência de distúrbios psiquiátricos entre os idosos considerados sem discernimento pode ser explicada ao considerar que na maioria dos casos, essas doenças são acompanhadas por perda de lucidez e autonomia comprometendo a função cognitiva.

Estudos mostram que os idosos com comprometimento da função cognitiva com graus variados de dependência ${ }^{14,17}$, por exigirem de suas famílias cuidados mais complexos e melhor estrutura física, econômica e psicossocial, tornamse o principal grupo a ser institucionalizado ${ }^{10,14,17}$.

Apesar dos resultados deste estudo contrariar tal hipótese, pois a maioria apresenta bom estado cognitivo; observa-se uma proporção significativa de idosos sem discernimento $(46,1 \%)$ indicando que a prevalência de doenças psiquiátricas pode ser um fator importante para institucionalização de alguns deles.

Apesar de a literatura afirmar que os idosos com disfunção cognitiva por apresentar em geral problemas crônicos de saúde e limitações funcionais; são os maiores usuários de polifarmá$\mathrm{cia}^{21}$. Loyola Filho ${ }^{19}$ mostrou um consumo menor de todas as classes de medicamentos entre 
esses idosos, dados também mostrados pelo presente estudo.

Acredita-se que essa subutilização de medicamentos se deve ao status cognitivo dos pacientes e, por conseguinte, maior suscetibilidade ao descumprimento do regime terapêutico implicando em prescrição de um menor número de medicamentos ${ }^{19}$.

As morbidades do grupo estudado justificam o perfil farmacoterapêutico e a média de consumo de cinco medicamentos por idoso, mostrado por outros trabalhos ${ }^{2-6,8,15,16,18,20}$. As semelhanças no perfil epidemiológico e de utilização de medicamentos mostrado por esses estudos parecem refletir problemas e necessidades comuns do grupo.

O maior consumo de medicamentos pelas mulheres, achado de outros estudos ${ }^{3,4,7,19}$, pode ser explicado pela idade mais avançada desse grupo, pois entre elas há uma maior proporção de indivíduos com idade superior a 70 anos. Essa hipótese pode ser ratificada pela análise da relação positiva entre o número de medicamentos consumidos e a idade, a qual mostrou aumento do consumo de medicamentos com seu avanço ${ }^{3}$ (Figuras 1 e 2).

A polifarmácia e a carência de acompanhamento farmacoterapêutico, situações encontradas no grupo estudado, podem aumentar a incidência de problemas relacionados a medicamentos (PRM) como erros de medicação, interações medicamentosas, prática de automedicação, dificuldade de adesão e surgimento de reações adversas ${ }^{3,4,6,7,9,15}$ comprometendo a qualidade da terapêutica.

Em idosos, tais problemas podem ainda ser agravados pelas limitações comuns da idade como dificuldade motora, visual, auditiva e de deglutição, pela redução da capacidade cognitiva e funcional, pela elevada prevalência de distúrbios psiquiátricos, pelo estado desmotivado e depressivo e ainda pelo baixo nível de instrução e de renda familiar ${ }^{6,14,16,17,20}$. No grupo estudado, acredita-se que a presença desses fatores influencia fortemente o surgimento de PRM, sobretudo o comprometimento da adesão ao esquema terapêutico.

A adesão constitui uma variável multifatorial sofrendo influência do estado de saúde do paciente, do acesso a medicamentos, da renda familiar, do grau de instrução, da presença e intensidade de reações adversas, do estado psicológico e psíquico e do surgimento de resultados benéficos com o tratamento $0^{6,12,16,20}$.

Nos institucionalizados a adesão é maior que em outros grupos de idosos em virtude da ad- ministração de medicamentos estar sob responsabilidade dos cuidadores e não do próprio idoso, tornando ausentes fatores como esquecimento e suspensão do uso ou alteração do esquema terapêutico por conta própria; comuns entre os não supervisionados por familiar, cuidador ou profissional de saúde.

Estudo realizado por Cintra et al. ${ }^{16}$ mostrou que os problemas com utilização e administração de medicamentos são minimizados quando os idosos são acompanhados por familiares e/ ou cuidadores. Segundo os autores a presença da família ou do cuidador é imprescindível para o cumprimento da terapia medicamentosa, pois o avanço da idade é acompanhado por aumento da dependência para executar atividades rotineiras como administração dos medicamentos ${ }^{16}$.

Como nas ILPI a responsabilidade de administração e de garantia de acesso aos medicamentos é da instituição e não do idoso, problemas de adesão e acesso a medicamentos, no grupo estudado, são minimizados. Ao contrário, idosos não institucionalizados podem apresentar problemas de acesso a medicamentos no serviço público de saúde ${ }^{16,23}$. Ainda assim, alguns institucionalizados, sobretudo os com discernimento, apresentam recusa em tomar seus medicamentos, descartando, cuspindo ou não engolindo os comprimidos; justificando a presença de problemas relacionados à adesão no grupo estudado.

Resultados mostram ainda que apesar de $61,7 \%$ dos idosos relatarem dificuldade de acesso a medicamentos como um fator limitador da adesão, grande parte dos medicamentos prescritos e utilizados pelo grupo estava presente na instituição no momento da pesquisa.

Porém, vale ressaltar que tal disponibilidade é variável ao longo do tempo e o dado obtido é autorreferido, sendo que tanto idosos como profissionais queixaram-se de falta de medicamentos apontando a compra com recursos próprios, do idoso ou da instituição, como a alternativa para garantir o acesso e minimizar tal problema. Como tal dado foi obtido a partir de relatos dos idosos ou dos cuidadores, há a possibilidade do mesmo ter sido superestimado, em virtude da observação de que muitos deles são poliqueixosos em relação à situação precária das instituições em que vivem ou trabalham.

Apesar da pequena prevalência de problemas que comprometem a adesão entre os idosos participantes do presente estudo, resultados sugerem que a dificuldade de acesso, o número elevado de medicamentos, a presença de efeitos adversos e as limitações comuns da idade são fato- 
res importantes que podem comprometer a adesão à prescrição médica, corroborando dados da literatura ${ }^{6,12,20}$.

Além desses fatores a elevada prevalência de doenças psiquiátricas, o frequente estado desmotivado e depressivo e o nível de escolaridade podem explicar o desconhecimento de alguns idosos com discernimento a respeito do esquema terapêutico utilizado, pois sabe-se que o entendimento e o comprometimento com a farmacoterapia representam importantes fatores para garantir a adesão ${ }^{6}$.

Apesar da influência direta do nível de instrução na compreensão e conseguinte adesão ao tratamento ${ }^{4,6,8,20}$, neste estudo, o nível de informação dos idosos não apresentou relação direta com os dados de escolaridade em ambos os sexos. Acredita-se que como nas ILPI a administração de medicamentos é responsabilidade dos cuidadores, não há preocupação em orientar o idoso quanto à prescrição médica.

Igualmente, vários estudos têm mostrado forte influencia da disponibilidade de medicamentos e do nível socioeconômico do usuário na adesão à farmacoterapia ${ }^{6,24}$.

Dados da Organização Mundial de Saúde (citados por Rozenfeld ${ }^{7}$ ) mostram que $25 \%$ da população mundial estão sem assistência farmacêutica completa, não tendo acesso a medicamentos essenciais ou o tendo de maneira limitada ${ }^{7}$.

Este estudo mostrou que o grupo avaliado não encontrava dificuldade de acesso a medicamentos, pois além de serem abastecidas pela rede de Atenção Básica do Sistema Único de Saúde (SUS); as ILPI em sua maioria por serem de caráter filantrópico têm o abastecimento de medicamentos garantido por meio de doações da comunidade e de recursos obtidos através de eventos beneficentes, sendo pequeno o gasto com medicamentos não disponibilizados dessas maneiras.

Porém, a falta de medicamentos de distribuição gratuita, mesmo pequena (12,3\%), aliada à baixa renda mensal dos idosos, em sua maioria aposentados com um rendimento inferior a dois salários-mínimos, são fatores importantes que limitam o acesso e, por conseguinte, para adesão ao tratamento prescrito ${ }^{6,24}$.

Fatores como dificuldade de acesso às informações dos prontuários, em geral com dados ausentes ou incompletos; influencia do estado psicológico do idoso nas informações por ele fornecidas, numero elevado de idosos com estado cognitivo comprometido em diferentes graus, ausência de farmacêuticos nas instituições para organizar os dados referentes a medicamentos; podem influenciar diretamente os resultados deste estudo, permitindo concluir a necessidade da realização de mais pesquisas com essa população.

No entanto, resultados obtidos permitem concluir que um acompanhamento farmacoterapêutico efetivo; a inserção de ações como incentivo à prática de atividades físicas para manutenção da capacidade funcional e de ações educativas que promovam o conhecimento sobre a farmacoterapia são imprescindíveis como forma de estimular o autocuidado, a adesão ao esquema terapêutico e, por conseguinte, melhoria da qualidade de vida dos idosos.

Faz-se necessário ainda, promover maior integração dos serviços do Sistema Único de Saúde e das Instituições de Longa Permanência de Idosos a fim de otimizar o atendimento aos idosos que ali vivem e o próprio funcionamento dessas instituições sociais.

Além disso, ações educativas no sentido de aumentar a formação e a capacitação de recursos humanos devem ser promovidas como forma de melhorar a assistência à saúde dos idosos proporcionando-lhes assistência integral e especializada. Sugere-se para isso, a incorporação dessas e de outras ações nas Políticas Públicas de Saúde como forma de minimizar o impacto do envelhecimento populacional sobre a qualidade de vida e promover um envelhecimento saudável com racionalização dos recursos necessários ${ }^{1,11}$.

\section{Colaboradores}

MPF Oliveira e MRCG Novaes participaram igualmente de todas as etapas de elaboração do artigo.

\section{Agradecimentos}

À Direção das Instituições de Longa Permanência, a seus profissionais e pacientes, pela valiosa colaboração para realização deste estudo. 
Referências

1. Chaimowicz F. A saúde dos idosos brasileiros às vésperas do século XXI: problemas, projeções e alternativas. Rev Saude Publica 1997; 31(2):184-200.

2. Danilow MZ, Moreira ACS, Villela CG, Barra BB, Novaes MRCG, Oliveira MPF. Perfil epidemiológico, sociodemográfico e psicossocial de idosos institucionalizados do Distrito Federal. Com Ciências Saúde 2007; 18(1):9-16.

3. Flores LM, Mengue SS. Uso de medicamentos por idosos em região do sul do Brasil. Rev Saude Publica 2005; 39(6):924-929.

4. Ribeiro AQ, Rozenfeld S, Klein CH, Cesar CC, Acurcio FA. Inquérito sobre uso de medicamentos por idosos aposentados, Belo Horizonte, MG. Rev Saude Publica 2008; 42(4):724-732

5. Oliveira MPF. Assistência Farmacêutica a idosos institucionalizados do Distrito Federal [dissertação]. Brasília (DF): Universidade de Brasília; 2008.

6. Rocha CH, Oliveira APS, Ferreira C, Faggiani FT, Schroeter G, Souza ACA, DeCarli GA, Morrone $\mathrm{FB}$, Werlang MC. Adesão à prescrição médica em idosos de Porto Alegre, RS. Cien Saude Colet 2008 13(Supl.):703-710.

7. Rozenfeld S. Prevalência, fatores associados e mau uso de medicamentos entre os idosos: uma revisão. Cad Saude Publica 2003; 19(3):717-724.

8. Blanski CRK, Lenardt MHA. A compreensão da terapêutica medicamentosa pelo idoso. Rev Gaúcha Enferm 2005; 26(2):180-188.

9. Medeiros-Souza P. Santos-Neto LL, Kusano LTE Pereira MG. Diagnóstico e controle da polifarmacia no idoso. Rev Saude Publica 2007; 41(6):1049-1053.

10. Perlini NMOG, Leite MT, Furini AC. Em busca de uma instituição para a pessoa idosa morar: motivos apontados por familiares. Rev Esc Enferm USP 2007; 41(2):229-236.

11. Creutzberg M, Gonçalves LHT, Sobottka EA, Ojeda BS. Long-term care institutions for elders and the health system. Rev Latino-am Enfermagem 2007; 15(6):1144-1149.

12. Chaudri NA. Adherence to Long-term Therapies Evidence for Action. Ann Saudi Med 2004; 24(3):221 222

13. Machuca M, Fernández-Llimós F, Faus MJ. Método Dáder. Guia de Seguimento Farmacoterapêutico. 3a Edição. Granada: GIAF-UGR; 2003.

14. Araújo MOPH, Ceolim MF. Avaliação do grau de independência de idosos residentes em instituições de longa permanência. Rev Esc Enferm USP 2007; 41(3):378-385.
15. Castellar JI, Karnikowski MGO, Vianna LG, Nobrega OT. Estudo da Farmacoterapia Prescrita em a Idosos em Instituição Brasileira de Longa Permanência. Acta Med Port 2007; 20:97-105.

16. Cintra FA, Guariento ME, Miyasaki LA. Adesão medicamentosa em idosos em seguimento ambulatorial. Cien Saude Colet 2010; 15(Supl. 3):3507-3515.

17. Converso MER, Iartelli I. Caracterização e análise do estado mental e funcional de idosos institucionalizados em instituições públicas de longa permanência. J Bras Psiquiatr 2007; 56(4):267-272.

18. Correr CJ, Pontarolo R, Ferreira LC, Baptistão SAM. Riscos de problemas relacionados com medicamentos em pacientes de uma instituição geriátrica. Rev Bras Cienc Farm 2007; 43(1):55-62.

19. Loyola Filho AI. Influência da renda na associação entre disfunção cognitiva e polifarmácia: Projeto Bambuí. Rev Saude Publica 2008; 42(1):89-99.

20. Teixeira JJV, Cano FG, Sanches ACC, Carniel TA, Schneider DSLG. Inquérito farmacoepidemiológico de pacientes de meia idade e idosos em três comunidades de Cascavel, PR, Brasil. Verificação evolutiva do conhecimento terapêutico. Rev Bras Cienc Farm 2008; 44(2):297-303.

21. Nogueira SL, Ribeiro RCL, Rosado LEFPL, Franceschini SCC, Ribeiro AQ, Pereira ET. Fatores determinantes da capacidade funcional em idosos longevos. Rev Bras Fisioter 2010; 14(4):322-329.

22. Cruz AV, Fulone I, Alcalá M, Fernandes AA, Montebelo MI, Lopes LC. Uso crônico de diazepam em idosos atendidos na rede pública em Tatuí-SP. Rev Cienc Farm Básica Apl 2006; 27(3):259-267.

23. Lima-Costa MF, Barreto SM, Giatti L. Condições de saúde, capacidade funcional, uso de serviços de saúde e gastos com medicamentos da população idosa brasileira: um estudo descritivo baseado na Pesquisa Nacional por Amostra de Domicílios. Cad Saude Publica 2003; 19(3):735-743.

24. Paniz VMV, Fassa AG, Facchini LA, Bertoldi AD, Piccini RX, Tomasi E, Thumé E, Silveira FV, Rodrigues MA. Acesso a medicamentos de uso contínuo em adultos e idosos nas regiões Sul e Nordeste do Brasil. Cad Saude Publica 2008; 24(2):267-280.

Artigo apresentado em 18/10/2011

Aprovado em 19/01/2012

Versão final apresentada em 23/01/2012 